\title{
ANÁLISIS DE UNA COLECCIÓN DE GARRAPATAS (ACARI: ARGASIDAE, IXODIDAE) DEL NORTE ARGENTINO
}

\author{
OrTiZ, F, NAVA, S. ${ }^{2} \&$ Guglielmone, A. A. ${ }^{2}$
}

\begin{abstract}
RESUMEN
Se analizó una colección de garrapatas obtenidas de hospedadores domésticos y silvestres de Fornosa, Jujuy, Salta y Santiago del Estero (Argentina). Las especies de garrapatas determinadas fucron Amblyomma argentinae, Amblyomma cajenennse sensu lato. Amblyomma neumanni, Amblyomna ovale, Ammbtyomna parvitarsum. Amblyomma parvum, Amblyomma rigrinum, Haemaphysalts juxtakochi, Ixodes luciae, Lxodes pararicinus, Ornithodoros rostratus. Ornithodoros sp.. Otobits megnini y Rhipicephaltus sanguineus sensu lato. Las determinaciones de / huciae y del grupo R. sanguineus amplía el rango de sus distribuciones en la Argentina a la provincia de Jujuy. Se presenta a Mazama americana (Artiodactyla Cervidae), Penelope obscura (Galliformes: Cracidae), Agelaioides badius (Passeriformes: Icteridae) y Myioborus brunniceps (Passeriformes: Parulidae) como nuevos hospedadores de A. neumanni, II. juxtakochi, A, tigrimum e I. pararicinus, respectivamente.

Palabras clave: Argasidae. Ixodidae, hospedadores, Amblyomma, Haemaphysatis, Ixodes, Ornizhodoros, Olobius. Rhipicephalus, norte argentino
\end{abstract}

\section{SUMMARY \\ Analysis of a tick (Acari: Argasidae, Ixodidae) collection from wild and domestic hosts in northern Argentina.}

A collection of tichs from wild and domestic hosis from the provinces of Formosa, Jujuy, Salta y Santiago del Estero (Argentina) was analyzed. The species of ticks found were Amblyomma argentinae, Amblyomma cajenennse seusu lato, Amblyomma neumanni, Amblyomma ovale. Ammblyomma parvitarsum, Amblyonma parvum, Amblyomma tigrinum, Haemaphysalis juxlakochi, Ixodes laciae, Ixodes pararicinus. Ornithodoros rostratus, Ornithodoros sp., Otobius megnini and Rhipicephalus

\footnotetext{
1.- Conscjo Nacional de Investigaciones Cientificas y Técnicas, Instituto Biologia de la Altura. universidad Nacional de Jujuy. Av. Bolivia 1661. SS de Jujuy. Jujuy, Argentina. 2.- Instituto Nacional de Tecnologia Agropecuaria, Estación Experimental Agropecuaria Rafaela y Consejo Nacional de Investigaciones Cientificas y Téenicas, CC 22. (2300) Rafaela, Santa Fe. Manuscrito recibido el 18 de abril de 2011 y aceptado para su publicacion el 22 de junio de 2011 .
} 
sanguineus sensu lato. The findings of I. lnciae and $R$ sangtineus group increase their Argentinean distributions into the province of Jujuy. Mazama americana (Artiodactyla: Cervidae). Penelope obscara (Gallifonnes: Cracidae), Agelaioides badius (Passerifornes: Icteridae) y. Wyobonus branniceps (Passerifomes: Parulidae) were deternined as new hosts for A. neumannh, I., huxtahochi, A. tigrimum and $I$ pararicinus, respectively.

Key words: Argasidac. Ixodidac, hosts. Amblyomma, Haemaphralis, Ixodes, Omihodoros. Otobilus, Rhipicephalts. northern Argentina

\section{INTRODUCCIÓN}

Las garrapatas de las familias Argasidae e Ixodidae son reconocidas como vectores de microorganismo patógenos para los tetrápodos, incluido el hombre, además de provocar perjuicios por el parasitismo per se o la inoculación de toxinas (Guglielmone et al., 2004). Las garrapatas del norte argentino han sido motivo de estudios para numerosos investigadores desde principios del siglo $\mathrm{XX}$, pero aún existe un amplio espectro de información que debe ser analizado para alcanzar una comprensión global de la ecología de garrapatas que se distribuyen en dicha region de la Argentina. A continuación se presenta un análisis de una colección de garrapatas obtenidas en las provincias de Formosa. Jujuy, Salta y Santiago del Estero que incrementan el área de distribución de algunas especies y presenta nuevos hospedadores para otras.

\section{MATERIALES Y MÉTODOS}

Los datos utilizados en este trabajo fueron obtenidos a partir del examen de garrapatas sin determinación taxonomica depositadas en la colección del Instituto Biología de la Altura, Universidad Nacional de Jujuy,
Argentina. Las garrapatas fueron determinadas siguiendo a Boero (1957), Jones et al. (1972), Guglielmone \& Viñabal (1994) y Estrada-Peña et al. (2005). La clasificación taxonómica de los mamíferos y aves se realizo de acuerdo a los esquemas propuestos por Wilson \& Reeder (2005) y Dickinson (2003), respectivamente, con la excepción del genero Galea (Rodentia: Caviidae) que fue modificado recientemente por Dunnum \& Salazar-Bravo (2010). Los hospedadores y localidades se cotejaron con la compilación de registros de garrapatas del neotrópico de uno de los autores ( $\mathrm{AAG}$ ), la cuál está disponible para los interesados que la soliciten. Es necesario mencionar que estudios recientes han demostrado que Rhipicephalus sanguinezs y Amblyomma cajennense son en realidad complejos de especies (Szabo et al. 2005; Moraes-Filho et al. 2010; Labruna et al. 2011; Mastropaolo et al. 2011). Por lo tanto. en este trabajo ambos taxones serán considerados de esa manera

\section{RESULTADOS}

La información de las especies de gatrapatas, cantidad por estadios colectados, lugar, coordenadas, altitud, fecha de hallazgo y colector se presentan en el Cuadro 1 de 